\title{
Aggressive Deeskalation der adjuvanten Therapie beim HPV-assoziierten Oropharynxkarzinom. Reicht die Hälfte von allem?
}

\author{
Markus Hecht ${ }^{1}$ - Sabine Semrau ${ }^{1}$ \\ Online publiziert: 16. März 2020 \\ (c) Der/die Autor(en) 2020
}

Hintergrund Die Beeinträchtigung des Schluckens und der Salivation nach einer multimodalen Therapie von Patienten mit Kopf-Hals-Tumoren ist fraglos gravierend. Dies motiviert, über eine Verringerung der Behandlungsintensität bei prognostisch günstigen Ausgangssituationen nachzudenken. Gemeint sind nichtrauchende Patienten mit einem HPV-assoziierten Oropharynxkarzinom. Verschiedene Strategien werden derzeit parallel untersucht, beispielsweise geringere Dosen in der postoperativen und definitiven Situation, der gänzliche Verzicht auf eine postoperative Bestrahlung oder die Substitution von Cisplatin bei der Chemotherapie.

Patienten und Methode Gleich mehrere Stellschrauben wurden bei einer postoperativen Radiochemotherapie (RCT) innerhalb einer Phase-II-Studie gelockert, die an zwei Mayo-Kliniken durchgeführt wurde [1]. Bei diesen Patienten wäre nach den Leitlinien des NCCN formal eine übliche postoperative Radiotherapie (RT) oder RCT indiziert gewesen [2]. Die Patienten mit allenfalls kurzer Raucheranamnese und einem Oropharynxkarzinom p16+ sowie mit mindestens einem Risikofaktor (L1, Pn1, $\leq$ pT3-Tumor, $\geq$ pN2a nach AJCC 7. Auflage) erhielten aber eine postoperative Strahlentherapie mit nur 1,5 Gy BID bis $30 \mathrm{~Gy}$. Patienten, deren Lymphknotenmetastasen sich bereits extranodal ausgebreitet hatten, erhielten zweimal täglich $1,8 \mathrm{~Gy}$ als simultan integrierten Boost (SIB)

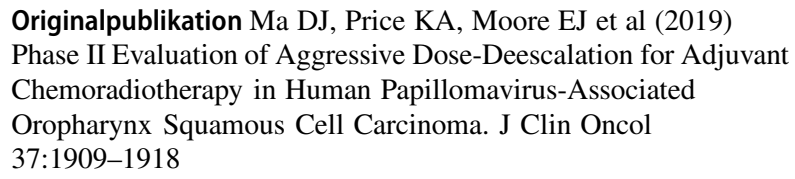

1 Universitäts-Strahlenklinik, Universitätsklinikum Erlangen, Universitätsstraße 27, 91054 Erlangen, Deutschland auf die extranodalen Manifestationen. Sonst umfasste das 30Gy-CTV bei sämtlichen Patienten das Tumorbett und ausschließlich die ipsilateralen Lymphknoten der Level II bis IV mit optionaler Erweiterung auf Level IB, V und die retropharyngealen Lymphknotenstationen. Ausnahmen waren Fälle mit Befall des weichen Gaumens und des Zungengrunds. Hier wurden ebenfalls die kontralateralen Lymphknoten der Level II bis IV bestrahlt. Als dritte Form der Deeskalation wurde die Chemotherapie auf Docetaxel umgestellt und die Einzel- und Gesamtdosis am Behandlungstag 1 und 8 stark gedrosselt auf $15 \mathrm{mg} / \mathrm{m}^{2}$. Mutmaßlich war die Therapie so konzipiert, dass im Falle eines Rezidivs noch eine voll dosierte Salvage-RCT durchzuführen war.

Ergebnisse In den zwei Kohorten mit 36 Patienten ohne extrakapsulären Lymphknotenbefall (30 Gy) und 43 Patienten mit extrakapsulärer Tumorbeteiligung (36Gy als SIB), die im Mittel eine Dosis von 24,8 Gy an den Mm. constrictores und 17,8 Gy an den Glandulae parotideae erhalten hatten, entwickelten erwartungsgemäß nur wenige Patienten eine Grad-3-Toxizität inform einer enoralen Mukositis oder Pharyngitis, und dies trotz hyperfraktionierter und akzelerierter Bestrahlung. Nach zwei Jahren hatte kein Patient höhergradige Spätfolgen. Kein Patient benötigte einen Monat nach Therapieende noch eine PEG. Bei Patienten ohne extrakapsulären Lymphknotenbefall war die lokale Kontrolle $100 \%$ und bei solchen mit extrakapsulärem Tumorbefall 93\% nach zwei Jahren. Alle Items der Lebensqualität erreichten das präradiotherapeutische Niveau oder verbesserten sich noch darüber hinaus.

Schlussfolgerung der Autoren Im Vergleich zu historischen Kontrollen konnten die beschriebenen Maßnahmen der Therapiedeeskalation eine deutlich bessere Lebensqualität erhalten, ohne dass Einschränkungen in der Tumorkontrolle bemerkt wurden. 


\section{Kommentar}

Die Behandlungsergebnisse aus den zwei Mayo-Kliniken klingen verlockend, bei einem Patienten mit einem HPVpositiven Oropharynxkarzinom zunächst eine Tumortonsillektomie vorzunehmen, dann mit einem reduzierten Volumen und einer reduzierten Dosis zu bestrahlen und simultan dazu mit einer atoxischen Chemotherapie zu behandeln. Die Resultate zur Krankheitskontrolle - zumindest bei Patienten ohne lymphknotenüberschreitendes Tumorwachstum sind sehr gut. Es gibt keine Einbußen an Lebensqualität, die nicht schon durch die Tumorresektion hervorgerufen worden sind. Reicht diese Information zum jetzigen Zeitpunkt bereits aus, um das Tumorboard von einer gewebeschonenden, enoralen Resektion mit einer anschließenden dosisreduzierten adjuvanten Radiochemotherapie zu überzeugen? Bei Weitem nicht! Die detaillierte Betrachtung der Ergebnisse bei den 80 Studienpatienten lässt einen bei drei Problemen hängen.

1. Es bedurfte sicher einer kenntnisreichen Patientenselektion und qualitativ exzellenten operativen Resektion, um die gezeigten herausragenden Ergebnisse zu erzielen. Es wurden nur Patienten in die Studie aufgenommen, die eine komplette Tumorresektion mit entsprechender Schnellschnittdiagnostik erhalten hatten. Patienten mit Tumoren, die in selbiger Sitzung nachreseziert werden mussten, was in der breiten Routineversorgung ja der Alltag ist, oder T4Tumoren entwickelten Rezidivraten in $15 \%$ der Fälle und eignen sich somit selbst nach R0-Resektion nicht. Diese Rezidivrate ist verglichen mit den Ergebnissen einer RCT zu hoch [3, 4]. Sie werden in der nächstens angestrebten Phase-III-Studie ausgeschlossen sein.

2. Der zweite Punkt, der nicht ausreichend thematisiert werden konnte, sind die Einbußen an Lebensqualität nach der Resektion im Vergleich zur primären voll dosierten RCT. Die 2019 veröffentlichte ORATOR-Studie zeigte für T1/2-Tumoren des Oropharynx langfristig eine bessere Lebensqualität nach einer konservativen Behandlung, verglichen mit einer primären transoralen Laserresektion, also dem Verfahren mit dem potenziell geringsten Gewebeverlust im Vergleich zu allen anderen Resektionsverfahren [5]. Es ist davon auszugehen, dass in der vorliegenden Arbeit die Beeinträchtigung der Schluckfunktion eher unterschätzt wurde.

3. Zudem muss betont werden, dass es sich um eine Studie an lediglich 80 Patienten handelt, bei der es darauf ankam, einen ersten Eindruck von der Rezidivhäufigkeit und vorrangig der zu erwartenden Lebensqualität zu erhalten. Es wurden auch Patienten eingeschlossen, die hierzulande keine Radiotherapie wegen des relativ günstigen Risikoprofils erhalten hätten. Die Gruppe von 43 Patienten mit extranodalem Tumorwachstum ist dann doch recht klein, sodass auf einen kontrollierten Vergleich mit der Standardtherapie an einer größeren Patientenzahl nicht verzichtet werden darf. Erinnert sei nur an die Tatsache, dass bei einer definitiven Radiochemotherapie der Ersatz von Cisplatin durch Cetuximab beim HPV-assoziierten Oropharynxkarzinom entgegen aller Erwartung zu einer um 7\% schlechteren lokoregionären Kontrolle nach 5 Jahren und zu einem $6 \%$ und $8 \%$ schlechteren Gesamtüberleben nach 2 bzw. 5 Jahren führte $[3,4]$.

Etwas ist aber anders bei dieser initial stark gedrosselten postoperativen Therapiedichte im Vergleich mit einer definitiven RCT und damit sehr interessant. Man hätte hierbei die Option einer vollumfänglichen Salvage-Therapie, die eventuell nur bei wenigen Patienten erforderlich ist, sofern diese keine Fernmetastasen entwickelten. In der Phase-IIStudie kam es ja bei 3 Patienten zu einem isolierten Lokalrezidiv. Leider erfahren wir von deren Krankheitsfortschritt nichts. Es wird entscheidend sein zu wissen, wie damit bei einer größeren Population umgegangen wird und was dies für das Überleben der betroffenen Patienten bedeutet. Letztlich wird es doch darum gehen, ob sich das Therapiekonzept der gedrosselten Initialtherapie mit Inkaufnahme einer höheren Lokalrezidivrate und der Option einer Rettungstherapie zugunsten einer deutlich verbesserten Lebensqualität in der Praxis durchsetzen kann. Das ist die spannendste aller Fragen an dieser Phase-III-Studie.

Markus Hecht und Sabine Semrau, Erlangen

Funding Open Access funding provided by Projekt DEAL.

Interessenkonflikt M. Hecht und S. Semrau geben an, dass kein Interessenkonflikt besteht.

Open Access Dieser Artikel wird unter der Creative Commons Namensnennung 4.0 International Lizenz veröffentlicht, welche die Nutzung, Vervielfältigung, Bearbeitung, Verbreitung und Wiedergabe in jeglichem Medium und Format erlaubt, sofern Sie den/die ursprünglichen Autor(en) und die Quelle ordnungsgemäß nennen, einen Link zur Creative Commons Lizenz beifügen und angeben, ob Änderungen vorgenommen wurden.

Die in diesem Artikel enthaltenen Bilder und sonstiges Drittmaterial unterliegen ebenfalls der genannten Creative Commons Lizenz, sofern sich aus der Abbildungslegende nichts anderes ergibt. Sofern das betreffende Material nicht unter der genannten Creative Commons Lizenz steht und die betreffende Handlung nicht nach gesetzlichen Vorschriften erlaubt ist, ist für die oben aufgeführten Weiterverwendungen des Materials die Einwilligung des jeweiligen Rechteinhabers einzuholen.

Weitere Details zur Lizenz entnehmen Sie bitte der Lizenzinformation auf http://creativecommons.org/licenses/by/4.0/deed.de.

\section{Literatur}

1. Ma DJ, Price KA, Moore EJ et al (2019) Phase II evaluation of aggressive dose de-escalation for adjuvant chemoradiotherapy in hu- 
man papillomavirus-associated oropharynx squamous cell carcinoma. J Clin Oncol 37:1909-1918

2. NCCN Practical Guidelines in Oncology, Head and Neck Cancer, Version 3.2019-September 16,2019at NCCN.org

3. Gillison ML, Trotti AM, Harris J et al (2019) Radiotherapy plus cetuximab or cisplatin in human papillomavirus-positive oropharyngeal cancer (NRG Oncology RTOG 1016): a randomised, multicentre, non-inferiority trial. Lancet 393:40-50
4. Mehanna H, Robinson M, Hartley A et al (2019) Radiotherapy plus cisplatinorcetuximab in low-risk human papillomavirus-positive oropharyngealcancer (De-ESCALaTE HPV): an open-label randomisedcontrolledphase 3 trial. Lancet 393:51-60

5. Nichols AC, Theurer J, Prisman E et al (2019) Radiotherapy versus transoral roboticsurgeryand neck dissectionfororopharyngealsquamouscellcarcinoma (ORATOR): an open-label, phase 2, randomisedtrial. Lancet Oncol 20:1349-1359 